\title{
Differential effects of Age-of-Acquisition for concrete nouns and action verbs: Evidence for partly distinct representations?
}

\author{
Véronique Boulenger, Nathalie Décoppet, Alice Roy, Yves Paulignan, \\ \& Tatjana A. Nazir \\ Institut des Sciences Cognitives, Lyon, France
}

Address correspondence to:

Tatjana Nazir

Institut des Sciences Cognitives

UMR 5015 CNRS-UCBL

67, boulevard Pinel 69675 BRON Cedex, France

nazir@isc.cnrs.fr

Phone: (33) 437911255 


\begin{abstract}
There is growing evidence that words that are acquired early in life are processed faster and more accurately than words acquired later, even by adults. As neuropsychological and neuroimaging studies have implicated different brain networks in the processing of action verbs and concrete nouns, the present study was aimed at contrasting reaction times to early and later-acquired action verbs and concrete nouns, in order to determine whether effects of word learning age express differently for the two types of words. Our results show that while word frequency affected both types of words in the same way, distinct learning age effects were observed for action verbs and concrete nouns. A further experiment specified that this difference was observed for verbs describing actions belonging to the human motor repertoire, but not for verbs denoting actions past this repertoire (e.g. to neigh). We interpret these data within a recently emerging framework according to which language processing is associated with sensory motor programs.
\end{abstract}




\section{Introduction}

Since the work of Carroll and White (1973ab), there is growing evidence that the age at which a word is acquired during childhood affects performance throughout life. Words that are acquired early in life are processed faster and more accurately than later-acquired words, even as factors such as frequency of usage and word imageability are controlled (Barry, Morrison \& Ellis, 1997; Bonin, Fayol \& Chalard, 2001; Brown \& Watson, 1987; Brysbaert, 1996; Morrison, Ellis \& Quinlan, 1992; Stadthagen-Gonzalez, Bowers, \& Damian, 2004). Though researchers differ in the way they precisely account for the underlying mechanisms of age of acquisition (AoA hereafter; for a review see Ghyselinck, Lewis \& Brysbaert, 2004), there is a general agreement that these effects are the consequence of the way information is stored and accessed in the brain. According to recent theoretical accounts, AoA-effects could be characteristic of human learning in general and might reflect the gradual loss of plasticity in the learning system (Ellis \& Lambon-Ralph, 2000; Ghyselinck et al., 2004; Zevin \& Seidenberg, 2002). In support of this hypothesis, connectionist models that employ distributed representations can simulate genuine AoA-effects, provided that learning patterns are introduced at different points into training, learning of early and late patterns is cumulative and interleaved (Ellis \& Lambon-Ralph, 2000; Monaghan \& Ellis, 2002), and what is learned about one pattern does not entirely carry over to other patterns with which it shares structures (Zevin \& Seidenberg, 2002).

In the present study, we determined AoA-effects for action verbs and concrete nouns that were acquired at different periods during childhood. Verbs and nouns play different semantic and syntactic roles in language. While nouns are pointer to objects, persons, places, things, or ideas, verbs generally refer to actions or states of being. In apparent conformity with this word class partition, neuropsychological studies have provided evidence that processing of nouns and verbs can be independently disrupted, suggesting that the two types of words may be represented in partly distinct neural substrates (Pulvermüller, 1999a; Shapiro \& Caramazza, 2003). Hence, while damage to frontal, motor-processing areas in the left hemisphere correlates more often with difficulties in processing action verbs, damage to left temporal regions correlates with difficulties in processing nouns (Caramazza \& Hillis, 1991; Damasio \& Tranel, 1993; Daniele, Giustolisi, Silveri, Colosimo, \& Gainotti, 1994; Miceli, Silveri, Villa \& Caramazza, 1984; Shapiro, Pascual-Leone, Mottaghy, Gangitano, \& Caramazza, 2001). Electrophysiological (Preissl, Pulvermüller, Lutzenberger \& Birbaumer, 1995; Pulvermüller, Lutzenberger \& Preissl, 1999b) and neuroimaging studies with healthy subjects (Martin, Haxby, Lalonde, Wiggs \& Ungerleider, 1995; Perani, Cappa, Schnur, Tettamanti, 
Collina, Rosa \& Fazio, 1999; Petersen, Fox, Posner, Mintun \& Raichle, 1989) further support this view by showing that different neural regions are active during processing of the two types of words.

More recent studies add to this picture by specifying that words designating actions performed with different body parts activate the premotor and motor cortex in a somatotopic manner, similar to movement execution (Hauk, Johnsrude \& Pulvermüller, 2004a; Pulvermüller, 2005a; Tettamanti, Buccino, Saccuman, Gallese, Danna, Scifo, Fazio, Rizzolatti, Cappa, \& Perani, 2005). By describing the spatio-temporal activity patterns during processing of words with different action-related meaning Pulvermüller and colleagues (Pulvermüller, Shtyrov \& Ilmoniemi, 2005b) could further show that activity in these regions emerged within the first 200 ms following word onset, that is, almost immediately after first brain responses were observed in perisylvian language areas. Pulvermüller and colleagues interpreted these results within the frame of Hebbian learning principles (Hebb, 1949) following which action verbs could have meaning-related correlates in motor and premotor cortex because caretakers typically produce an action word to prompt children to execute the corresponding action (Goldfield, 2000). By contrast, words that represent objects (e.g., most of concrete nouns) could have meaning-related correlates in temporal visual areas because they often co-occur with visual percepts (Pulvermüller, 1999a, 2003). In other terms, aspects of word meaning could be laid down by specific corticocortical links that evolve through correlation learning (Hauk et al., 2004a; Hauk \& Pulvermüller, 2004b; Pulvermüller, 2005a) and this could explain the differential nature of neural representation for action verbs and concrete nouns.

If action verbs and concrete nouns do not make use of fully overlapping neural structures, AoA-effects could evolve differently for the two types of words (Bogka, Masterson, Druks, Fragkioudaki, Chatziprokopiou \& Economou, 2003; Bonin, Boyer, Méot, Fayol \& Droit, 2004; Colombo \& Burani, 2002; Morrison, Hirsh, \& Duggan, 2003). The purpose of the present study was to contrast reaction times to early and later-acquired action verbs and concrete nouns in order to determine whether the hypothesized difference in cortical representations could be noticed in participants' performance. Distinct AoA-dependent reaction time profiles (i.e., difference in reaction time slopes when plotted as a function of AoA) for action verbs and concrete nouns would support such a view.

To test this possibility, we first established AoA ratings for a set of action verbs and 
concrete nouns following a procedure that has been shown to be a valid index of real word learning age (Bonin, 2001; Morrison, Chappell, \& Ellis, 1997). As imageability of action verbs and concrete nouns generally differs, we also established ratings for word imageability. We then proposed a visual lexical decision task for two classes of target words and determined reaction time profiles as a function of rated AoA.

\section{Subjective ratings of AoA and word imageability}

\section{Participants}

Twenty-five volunteers (between 19 and 28 years old) performed AoA ratings and twentytwo volunteers (between 18 and 26 years old) imageability ratings. All participants were native French speakers and had intact or corrected vision.

\section{Materials}

Three hundred and six words (153 verbs and 153 nouns) were selected from the French lexical database "Lexique” (New, Pallier, Ferrand \& Matos, 2001). Verbs, all in infinitive form, denoted actions that could be performed with arms/hands, legs or mouth. Nouns, all in singular form, referred to imageable, concrete entities. Words that stand for nouns as well as verbs were excluded from the list. Verbs and nouns were matched one-to-one for relevant lexical variables (see Supplementary Methods 1).

\section{Procedure}

Following the procedure proposed by Gilhooly and Logie (1980), participants were asked to estimate the age at which they thought to have acquired the meaning of the words on a seven-point scale (with $1=$ [0-2 years old], 2 = [3-4 years old], etc., and 7 = [older than 13]). For word imageability estimations, participants were asked to evaluate the ease with which they could create an image of the concept referred to by the word. Rating was performed on a seven-point scale (with 0 = impossible and 6 = very easy). The total of 306 verbs and nouns were presented in booklets of four pages with random and different order for each participant.

\section{$\underline{\text { Results }}$}

AoA and imageability-scores were determined for each word item by averaging ratings over all participants. Table 1 plots results for verbs and nouns. The data show that action verbs were rated as acquired earlier than nouns and were significantly less imageable than nouns.

Table 1 (about here) 
A subset of 30 early and 30 later-acquired target verbs and 30 early and 30 later-acquired target nouns, for which word frequency and imageability were controlled, were selected from the initial pool of 306 items (see Appendix). As the initial pool of verbs and nouns differed considerably in terms of mean AoA and imageability, these variables could only be equated within, but not between the two groups of words (see Supplementary Methods 2). This is not problematic, however, as we were looking for differences in reaction time slopes between groups. To verify whether surface features such as the systematic ending of French verbs with “-er” may allow distinguishing the two groups of words prior to more advanced word processing, the same target words were also analyzed according to word frequency (low and high). If word frequency has comparable effects on reaction time for verbs and nouns, this proviso can be discarded. Unfortunately, when regrouped according to word frequency, the selected pool of target words could not be matched on all relevant lexical variables. For this reason, an additional subset of 152 low and higher frequency control-words was selected matched on all variables but word- and lemma frequency.

\section{Lexical decision experiment}

\section{Participants}

Twenty volunteers (10 women and 10 men), aged from 22 to 29 years old, participated in the lexical decision task. All were native French speakers and had normal or corrected to normal vision. Right-handedness was determined with the Edinburgh inventory (scores between 0.63 and 0.94; Oldfield, 1971). None of the participants took part in the previous ratings of AoA or imageability.

\section{$\underline{\text { Materials }}$}

The experimental stimuli consisted of the entire set of 306 words described earlier. Furthermore, 306 pseudo-words, constructed by changing one letter from real nouns or real verbs, were added to perform the lexical decision task. Hence, pseudo-words were either “pseudo-nouns” or "pseudo-verbs” and were all pronounceable. Pseudo-words were matched to words for lexical variables (see Supplementary Methods 1). We also carefully matched verbs and pseudo-verbs for endings, such that as many verbs as pseudo-verbs (130 out of 153) ended with "-er", to prevent it from serving as discriminating feature. All items were presented in lower case. 


\section{Procedure}

Stimuli were displayed at the center of the screen of a Macintosh monitor, using Psyscope software. A central fixation cross was presented for $500 \mathrm{~ms}$. The stimulus appeared immediately following the offset of the fixation cross and remained on the screen until participant indicated by a button press whether the stimulus was a word or not. Participants were instructed to respond as quickly and accurately as possible. Stimulus presentation was randomized and a short break occurred half way through the experiment. A training phase consisting of 10 words and 10 pseudo-words (different from the experimental stimuli) preceded the experiment. Accuracy and response latencies were recorded.

\section{$\underline{\text { Results }}$}

Trials with reaction times below or above 2.5 standard deviations from individual means (calculated over the entire set of 306 words) were excluded from the analysis (a total of 2.25\% trials). Accuracy and response latencies for nouns, verbs, pseudo-nouns and pseudo-verbs were first calculated individually and were then averaged over all participants.

\section{a) For the set of 306 words}

A first analysis of variance by subject (ANOVA) over the entire set of 306 words and 306 pseudo-words revealed significant longer reaction times for pseudo-words than for words (677 ms vs. 601 ms respectively, $[\mathrm{F}(1,19)=46.692 ; \mathrm{p}<.0001])$. Furthermore, despite the strict control for word-, bigram-, and trigram frequency, mean reaction times for nouns (611 ms) were significantly longer than for verbs $(591 \mathrm{~ms}$; $[\mathrm{F}(1,19)=8.856 ; \mathrm{p}=.0078])$. A similar pattern was found for the percentage of errors $(4.41 \%$ vs. $2.68 \%$ respectively; $[F(1,19)=$ 9.378; $\mathrm{p}=.0064])$. Simple regression analyses showed that, when reaction times were plotted as a function of AoA, the slope parameter and coefficient of determination $\left(\mathrm{R}^{2}\right)$ were greater for nouns (slope $=24.17, \mathrm{R}^{2}=.3247, \mathrm{p}<.0001$ ) than for verbs (slope $=15.39, \mathrm{R}^{2}=.1833, \mathrm{p}<$ .0001). Plotted as a function of word- and lemma frequency, however, the difference between the two groups of words was mainly on axis intercept (word frequency: slope $=-19.55, \mathrm{R}^{2}=$ $.2584, \mathrm{p}<.0001$ for nouns, and slope $=-14.48, \mathrm{R}^{2}=.2142, \mathrm{p}<.0001$ for verbs; lemma frequency: slope $=-12.74, \mathrm{R}^{2}=.1389, \mathrm{p}=.0014$ for nouns, and slope $=-15.81, \mathrm{R}^{2}=.1935, \mathrm{p}$ $=.0025$ for verbs). Finally, when reaction times were plotted as a function of imageability, the slope parameter and coefficient of determination were also greater for nouns (slope $=-27.45$, $\mathrm{R}^{2}=.1925, \mathrm{p}<.0001$ ) than for verbs (slope $=-14.58, \mathrm{R}^{2}=.0508, \mathrm{p}=.0051$ ). 
To disentangle the effects of the different variables, we performed multiple regressions accompanied by hierarchical analyses using mean reaction time as dependent variable and seven independent variables: bigram and trigram frequency, number of syllables, number of letters, imageability, word frequency, and AoA. These analyses showed that while word frequency significantly predicted performance for both nouns and verbs, AoA was a significant predictor of reaction time only for nouns. Imageability did not explain additional variance in either case (for details see Supplementary Results).

b) For the subset of $2 * 60$ early and late acquired words

Figure 1a plots mean reaction time for the group of early and late acquired target words. Figure $1 \mathrm{~b}$ plots mean reaction time for the same items when organized as a function of word frequency, and Figure 1c plots the data for the subset of 152 control-words. The lower panel of Figure 1 (d-f) gives the corresponding scatterplots and simple regression analyses. Results show that whereas AoA-effects were obtained for nouns, no such effect was observed for verbs. The slope parameter and coefficient of determination $\left(\mathrm{R}^{2}\right)$ were about three times larger for nouns than for verbs (Figure 1d). By contrast, effects of word frequency were comparable for both word categories (Figure 1e and f). Given that word frequency had the same effect on verbs and nouns, differential AoA-effects cannot be attributed to factors that occur prior to word processing. Multiple regression analyses (see Supplementary Results) confirmed this conclusion by showing a significant effect of word frequency in all cases, but a significant effect of AoA only for target nouns. Imageability did not explain additional variance.

Figure 1 (about here)

\section{General discussion}

The present study shows that while word frequency has the same effect on lexical decision time for concrete nouns and action verbs, AoA has not. Reaction times to early-acquired concrete nouns are generally faster than to later-acquired nouns, whereas no significant effect of AoA is evident for action verbs. It is important to note here that AoA-effects for verbs had been obtained in other studies, especially when using tasks that required word production (e.g., word or picture naming, Bogka et al., 2003 for English and Greek; Bonin et al., 2004 for French; Colombo \& Burani, 2002 for Italian; Morrison et al., 2003 for English). Hence, the present results should not be interpreted as demonstrating that there is no AoA-effect on performance for verbs. Effects of AoA may show up differently depending on task requirements, because different tasks are probing different aspects of word processing (e.g. 
phonological output lexicon in word production tasks vs. input stages in lexical decision tasks; Ghyselink et al., 2004). What we illustrate here is simply that with the same task and participants, estimated age of word learning affects performance differently for action verbs and concrete nouns. This difference is suggestive of the assumption that at some levels of processing, the way in which information is stored and accessed in the brain may differ for the two types of words.

As mentioned in the introduction, cortical motor regions are activated during processing of action-related language (Boulenger, Roy, Paulignan, Déprez, Jeannerod \& Nazir, in press; Buccino, Riggio, Melli, Binkofski, Gallese \& Rizzolatti, 2005; Hauk et al., 2004ab; Oliveri, Finocchiaro, Shapiro, Gangitano, Caramazza, \& Pascual-Leone, 2004; Pulvermüller et al., 2005b; Pulvermüller, Hauk, Nikulin, \& Ilmoniemi, 2005c; Shtyrov et al., 2004; Tettamanti et al., 2005). In a recent study, we could show that this language-related motor activity is significant to a point that it can interfere with the concurrent execution of overt motor behavior (Boulenger et al., in press). Hence, fine-grained analyses of movement kinematics revealed that, within the first $200 \mathrm{~ms}$ following word/movement onset, encoding of (arm) action verbs interfered with the concurrent execution of an arm reaching movement, in that it delayed the wrist acceleration peak (relative to nouns with no specific motor associations) by approximately $20 \mathrm{~ms}$. We interpreted this finding in terms of competition between motor and language tasks for common resources, thus supporting the view that neural representation of action words involves cortical motor regions (Buccino et al. 2005; Hauk et al., 2004ab; Pulvermüller, 2005a). On this background, we speculate that the absence of AoA-effects for action verbs in our present study may result from a failure to capture real learning-age by subjective AoA-estimations of the verbal form of verbs because the organization of neural representation for action verbs may also depend on developing motor behavior. In other terms, rated AoA did not affect performance for action verbs because our early and lateracquired items may not actually differ in learning-age if this motor component were properly controlled.

To substantiate this claim, we conducted an additional lexical decision experiment using verbs that do not contain such a motor component. In line with Buccino et al. (Buccino, Lui, Canessa, Patteri, Lagravinese, Benuzzi, Porro and Rizzolatti, 2004; see also Mason, Banfield and Macrae, 2004) who showed that distinct brain regions are active during observation of motor actions common to humans and animals (i.e. biting) and motor actions that are specific to animals (i.e. barking), we hypothesize that AoA-effects for verbs may become evident 
more readily if we use verbs that describe actions that do not belong to the human motor repertoire. Our second experiment thus included early and late acquired verbs designating actions, which were either specific to animals (i.e. neigh; $n=18$ ) or specific to humans (i.e. knead; n=18; see Supplementary Methods 3 for experimental details). The results of this experiment are given in Figure 2. Though this data should be interpreted with caution as it is based on a restricted number of words, it replicates the results of the first experiment in showing no effect of rated AoA for verbs denoting actions belonging to the human motor repertoire. However, for verbs denoting actions that do not belong to this repertoire, rated AoA affected performance in much the same way as it did for concrete nouns.

Figure 2 (about here)

\section{Conclusion}

The result of our first experiment shows that rated AoA has a different effect on lexical decision for concrete nouns and action verbs. This result thus lends credibility to the view that the two types of words may be represented in partly distinct neural substrates (Pulvermüller, 1999a; Shapiro \& Caramazza, 2003). The result of our second experiment further specifies that these differential effects occur only for verbs that describe actions belonging to the human motor repertoire. If we accept as general rule that AoA affects performance (Ellis \& Lambon-Ralph, 2000; Ghyselinck et al., 2004; Zevin \& Seidenberg, 2002), the absence of AoA-effects for verbs denoting human actions indicates that subjective estimations of the age at which the verbal form of these words were acquired may not capture real learning-age. The failure of capturing learning-age for these verbs by means of subjective estimations may simply be due to difference in the ease of this estimation for nouns and action verbs (e.g., it could be more difficult to rate the age at which we acquired the verbal form of action verbs than of concrete nouns). Alternatively, however, this difference may result from existing links between language processes and sensory and motor programs (Boulenger et al., in press; Lieberman, 2002; Martin et al., 1995; Pulvermüller, 1999a, 2005a; Tettamanti, et al., 2005). If the neural representations of verbs that describe human actions involve cortical motor regions, the age at which this representation sets may depend on developing motor behavior and may not be captured by estimations of AoA of the verbal form only.

\section{Authors'note}

We would like to thank three anonymous reviewers for helpful comments on an earlier version of this article.

Tatjana A. Nazir is a member of the Marie Curie Research and Training Network: Language 
and Brain (RTN: LAB) funded by the European Commission (MRTN-CT-2004-512141) as part of its "Sixth Framework Program”. 


\section{Appendix}

Target words used in Experiment 1

\begin{tabular}{|c|c|c|c|c|c|c|c|c|c|}
\hline & NOUNS & LETT & SYLL & FQ & LEM & BIGR & TRIG & AoA & IMAG \\
\hline \multirow{30}{*}{ 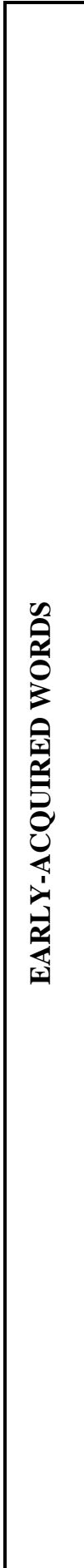 } & algue & 5 & 1 & 1,81 & 14,91 & 1433,2 & 140,62 & 4 & 5,23 \\
\hline & alouette & 8 & 3 & 1,71 & 3,1 & 2139,55 & 356,21 & 4,04 & 3,36 \\
\hline & bâtiment & 8 & 3 & 19,26 & 45,91 & 2565,18 & 807,14 & 4,2 & 5,00 \\
\hline & bordure & 7 & 2 & 8,65 & 9,68 & 3814,2 & 392,42 & 4,08 & 3,14 \\
\hline & cabine & 6 & 2 & 16,35 & 20,03 & 2548,7 & 341,33 & 3,92 & 4,14 \\
\hline & casier & 6 & 2 & 3,16 & 6,84 & 5375,81 & 328,88 & 4,08 & 4,32 \\
\hline & caverne & 7 & 2 & 4,9 & 9,09 & 2999,25 & 412,82 & 3,72 & 4,86 \\
\hline & cerisier & 8 & 3 & 1,68 & 2,91 & 6076,53 & 479,05 & 4,04 & 5,55 \\
\hline & dindon & 6 & 2 & 0,94 & 1,42 & 4158,66 & 43,2 & 4,08 & 5,00 \\
\hline & étable & 6 & 2 & 5,39 & 7,2 & 4094,23 & 1665,8 & 3,92 & 4,32 \\
\hline & étagère & 7 & 3 & 4,74 & 8,64 & 3647,75 & 1353,76 & 3,92 & 5,55 \\
\hline & flocon & 6 & 2 & 1,1 & 5,1 & 1898,56 & 177,65 & 3,16 & 5,27 \\
\hline & grenier & 7 & 2 & 13,68 & 17 & 3922,9 & 612,65 & 3,6 & 4,68 \\
\hline & hélice & 6 & 2 & 4,42 & 5,94 & 1347,87 & 184,38 & 4,12 & 5,18 \\
\hline & hibou & 5 & 2 & 1,48 & 1,96 & 1129,82 & 70,6 & 2,76 & 5,64 \\
\hline & hotte & 5 & 1 & 1,77 & 1,93 & 4705,12 & 883,37 & 3,12 & 4,41 \\
\hline & igloo & 5 & 2 & 0,03 & 0,13 & 408,86 & 0,34 & 3,6 & 5,77 \\
\hline & koala & 5 & 3 & 0,06 & 0,06 & 830,73 & 19,6 & 4,04 & 5,55 \\
\hline & limace & 6 & 2 & 1,97 & 3,13 & 2008,57 & 195,68 & 3,08 & 5,45 \\
\hline & mairie & 6 & 2 & 10,16 & 11,1 & 7211,44 & 1712,29 & 4,2 & 5,27 \\
\hline & moquette & 8 & 2 & 7,97 & 8,84 & 2650,77 & 339,62 & 3,12 & 4,82 \\
\hline & panda & 5 & 2 & 0,06 & 0,06 & 9829,56 & 490,58 & 3,32 & 5,77 \\
\hline & panneau & 7 & 2 & 12,16 & 24,26 & 7099,81 & 527,75 & 3,24 & 5,32 \\
\hline & prairie & 7 & 2 & 9,29 & 20,26 & 6623,51 & 663,49 & 3,6 & 5,14 \\
\hline & préau & 5 & 2 & 1,97 & 2,87 & 3854,63 & 868,27 & 3,16 & 4,18 \\
\hline & rosier & 6 & 2 & 1,16 & 3,19 & 3269,42 & 240,26 & 4,08 & 5,09 \\
\hline & ruche & 5 & 1 & 1,97 & 3,2 & 1443,66 & 443,07 & 3,92 & 5,18 \\
\hline & vaisseau & 8 & 2 & 6,16 & 14,93 & 5042,73 & 1769,35 & 3,8 & 4,32 \\
\hline & vipère & 6 & 2 & 2,06 & 3,32 & 3307,5 & 122,96 & 3,76 & 5,09 \\
\hline & virage & 6 & 2 & 5,94 & 8,68 & 3063,64 & 336,67 & 3,76 & 5,09 \\
\hline \multirow{6}{*}{ 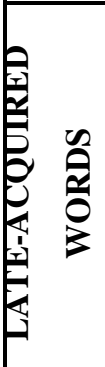 } & auberge & 7 & 2 & 9,71 & 12,32 & 3713,96 & 124,84 & 4,8 & 4,77 \\
\hline & baobab & 6 & 3 & 0,42 & 0,65 & 761,13 & 58,97 & 6 & 4,00 \\
\hline & barreau & 7 & 2 & 2,87 & 12,26 & 5837,49 & 586,01 & 4,44 & 4,82 \\
\hline & bécane & 6 & 2 & 2,1 & 2,58 & 2296,35 & 157,76 & 5,56 & 4,05 \\
\hline & bélier & 6 & 2 & 0,55 & 1,26 & 2716,51 & 338,99 & 4,68 & 5,18 \\
\hline & buffet & 6 & 2 & 12,19 & 13,54 & 840,36 & 89,52 & 4,6 & 4,91 \\
\hline
\end{tabular}




\begin{tabular}{|c|c|c|c|c|c|c|c|c|}
\hline canoë & 5 & 3 & 1,29 & 1,52 & 4856,14 & 159,17 & 4,96 & 5,50 \\
\hline avenue & 6 & 3 & 26,06 & 32,45 & 4485,24 & 998,91 & 4,8 & 4,50 \\
\hline cèdre & 5 & 1 & 1,84 & 2,87 & 3116,88 & 162,01 & 6,6 & 4,05 \\
\hline chalet & 6 & 2 & 3,74 & 4,42 & 4486,8 & 1101,2 & 4,36 & 5,64 \\
\hline dalle & 5 & 1 & 8,81 & 18,65 & 6074,85 & 1304,89 & 5,8 & 3,64 \\
\hline donjon & 6 & 2 & 2,35 & 2,87 & 7228,84 & 704,43 & 4,56 & 4,95 \\
\hline estrade & 7 & 2 & 6,03 & 6,35 & 5243,93 & 1937,05 & 5 & 4,68 \\
\hline évier & 5 & 2 & 6,16 & 6,61 & 2050,94 & 152,42 & 4,32 & 5,00 \\
\hline gradin & 6 & 2 & 0,65 & 4,07 & 3693,48 & 679,36 & 4,96 & 4,82 \\
\hline hangar & 6 & 2 & 6,97 & 10,87 & 3741,31 & 277,73 & 5 & 4,59 \\
\hline hublot & 6 & 2 & 2,1 & 3,68 & 1019,74 & 184,32 & 5,36 & 5,00 \\
\hline hutte & 5 & 1 & 4,13 & 6,58 & 4998,79 & 902,25 & 5,08 & 4,82 \\
\hline kayak & 5 & 2 & 0,71 & & 341,03 & 8,43 & 5,48 & 4,82 \\
\hline mulet & 5 & 2 & 2,1 & 4,78 & 2882,33 & 103,16 & 5,28 & 4,73 \\
\hline muraille & 8 & 2 & 7,58 & 14 & 3830,59 & 848,09 & 5,16 & 4,73 \\
\hline rambarde & 8 & 2 & 1,32 & 1,58 & 1494,19 & 156,91 & 4,72 & 3,91 \\
\hline otarie & 6 & 3 & 0,39 & 0,74 & 2904,51 & 79,53 & 4,56 & 4,95 \\
\hline palier & 6 & 2 & 17,32 & 21,45 & 7333,03 & 366,41 & 5,84 & 3,05 \\
\hline peuplier & 7 & 3 & 2,48 & 7,48 & 4855,43 & 827,45 & 5,16 & 4,36 \\
\hline plumage & 7 & 2 & 1,55 & 1,87 & 3025,12 & 1303,71 & 4,52 & 3,91 \\
\hline podium & 6 & 2 & 0,58 & 0,64 & 3015,08 & 39,49 & 5,2 & 5,00 \\
\hline poutre & 6 & 1 & 3,29 & 9,1 & 14187,9 & 3905,49 & 4,32 & 5,05 \\
\hline ravin & 5 & 2 & 5,29 & 7,26 & 2428,27 & 185,25 & 5,08 & 4,41 \\
\hline $\begin{array}{l}\text { vitrine } \\
\text { MEAN }\end{array}$ & 7 & 2 & 11,42 & 20,29 & 4475 & 532,9 & 4,32 & 4,91 \\
\hline
\end{tabular}




\begin{tabular}{|c|c|c|c|c|c|c|c|c|c|}
\hline & VERBS & LETT & SYLL & FQ & LEM & BIGR & TRIG & AoA & IMAG \\
\hline \multirow{30}{*}{ 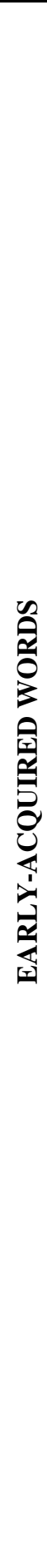 } & accrocher & 9 & 3 & 8,74 & 60,82 & 1369,06 & 389,72 & 3,20 & 3,82 \\
\hline & allumer & 7 & 3 & 11,42 & 74,6 & 3168,82 & 462,47 & 2,68 & 4,23 \\
\hline & appuyer & 7 & 3 & 13,32 & 91,2 & 2143,54 & 534,14 & 2,60 & 3,14 \\
\hline & aspirer & 7 & 3 & 2,77 & 22,6 & 3788,45 & 320,43 & 3,68 & 3,32 \\
\hline & balancer & 8 & 3 & 6,39 & 56,01 & 3114,93 & 480,56 & 3,00 & 4,36 \\
\hline & baver & 5 & 2 & 2,16 & 8,72 & 3015,87 & 421,85 & 2,32 & 4,68 \\
\hline & bricoler & 8 & 3 & 0,97 & 6,44 & 2228,52 & 162,77 & 3,56 & 4,00 \\
\hline & clouer & 6 & 2 & 1,55 & 12,4 & 2785,02 & 112,47 & 3,64 & 4,45 \\
\hline & cogner & 6 & 2 & 4,45 & 20,76 & 6319,57 & 267,86 & 3,52 & 4,05 \\
\hline & colorier & 8 & 3 & 0,32 & 3,19 & 5898,38 & 615,55 & 1,84 & 4,59 \\
\hline & copier & 6 & 2 & 3,39 & 28,39 & 6431,30 & 217,34 & 3,48 & 3,82 \\
\hline & découper & 8 & 3 & 3,81 & 25,47 & 3043,40 & 486,10 & 2,96 & 4,27 \\
\hline & fermer & 6 & 2 & 21,87 & 237,74 & 5747,26 & 840,83 & 1,88 & 3,91 \\
\hline & frotter & 7 & 2 & 4,94 & 27,86 & 4005,42 & 424,38 & 3,12 & 4,05 \\
\hline & gifler & 6 & 2 & 2,00 & 17,77 & 3077,20 & 156,16 & 3,56 & 4,59 \\
\hline & gommer & 6 & 2 & 0,97 & 10,67 & 6779,04 & 3145,50 & 2,68 & 4,64 \\
\hline & gratter & 7 & 2 & 4,94 & 24,48 & 4152,75 & 744,68 & 2,80 & 4,36 \\
\hline & griffer & 7 & 2 & 1,39 & 15,24 & 2372,61 & 141,21 & 2,60 & 4,00 \\
\hline & lécher & 6 & 2 & 3,52 & 14,47 & 2633,90 & 497,34 & 2,48 & 4,77 \\
\hline & mâcher & 6 & 2 & 2,06 & 7,3 & 2075,89 & 490,99 & 2,72 & 4,27 \\
\hline & nager & 5 & 2 & 5,58 & 19,41 & 2125,63 & 141,90 & 2,44 & 5,23 \\
\hline & nettoyer & 8 & 3 & 7,10 & 17,82 & 3057,08 & 528,39 & 3,20 & 4,36 \\
\hline & pincer & 6 & 2 & 2,35 & 28,69 & 3354,96 & 277,80 & 2,44 & 4,45 \\
\hline & remuer & 6 & 2 & 8,48 & 39,87 & 4602,33 & 243,26 & 3,48 & 4,05 \\
\hline & réparer & 7 & 3 & 9,58 & 17,36 & 4701,41 & 736,26 & 3,52 & 3,50 \\
\hline & rincer & 6 & 2 & 2,00 & 6,57 & 3439,04 & 260,57 & 3,40 & 3,86 \\
\hline & saler & 5 & 2 & 0,39 & 79,6 & 6306,76 & 471,06 & 3,68 & 4,45 \\
\hline & savonner & 8 & 3 & 0,77 & 2,44 & 3341,17 & 403,38 & 3,20 & 4,73 \\
\hline & téter & 5 & 2 & 1,16 & 4,83 & 3204,28 & 529,66 & 3,68 & 4,82 \\
\hline & trembler & 8 & 2 & 12,13 & 58,57 & 3043,47 & 363,00 & 3,48 & 4,14 \\
\hline \multirow{8}{*}{ 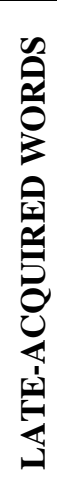 } & agiter & 6 & 3 & 6,68 & 58,63 & 4791,10 & 466,91 & 3,76 & 4,00 \\
\hline & saucer & 6 & 2 & 0,23 & 13,09 & 4550,69 & 304,90 & 4,92 & 3,59 \\
\hline & bêcher & 6 & 2 & 0,58 & 4,38 & 2056,37 & 444,84 & 5,48 & 3,64 \\
\hline & briser & 6 & 2 & 11,39 & 67,28 & 5882,28 & 656,68 & 4,48 & 4,00 \\
\hline & démolir & 7 & 3 & 2,39 & 7,79 & 2293,36 & 175,86 & 3,72 & 3,86 \\
\hline & \begin{tabular}{|l|} 
enterrer \\
\end{tabular} & 8 & 2 & 5,00 & 18,81 & 6643,37 & 1088,88 & 4,00 & 4,32 \\
\hline & épiler & 6 & 3 & 0,68 & 1,41 & 3463,74 & 210,76 & 6,04 & 4,45 \\
\hline & escalader & 9 & 4 & 2,32 & 13,57 & 2269,12 & 127,43 & 4,08 & 4,59 \\
\hline
\end{tabular}




\begin{tabular}{|c|c|c|c|c|c|c|c|c|}
\hline faucher & 7 & 2 & 2,06 & 9,79 & 3594,30 & 728,20 & 5,64 & 3,27 \\
\hline fouetter & 8 & 2 & 2,29 & 11,06 & 7192,99 & 407,18 & 4,72 & 4,27 \\
\hline hocher & 6 & 2 & 0,84 & 22,09 & 2700,71 & 490,91 & 4,76 & 3,45 \\
\hline jongler & 7 & 2 & 0,94 & 2,48 & 6503,16 & 289,80 & 3,84 & 4,55 \\
\hline masser & 6 & 2 & 1,10 & 130,07 & 7792,34 & 1151,23 & 4,72 & 4,00 \\
\hline noter & 5 & 2 & 21,97 & 172,28 & 5437,18 & 859,38 & 4,08 & 3,59 \\
\hline opérer & 6 & 3 & 12,06 & 79,69 & 3873,78 & 155,37 & 3,88 & 3,77 \\
\hline peler & 5 & 2 & 0,58 & 5 & 6442,66 & 163,33 & 5,16 & 4,45 \\
\hline pendre & 6 & 1 & 5,26 & 36,99 & 6505,33 & 994,96 & 4,4 & 4,09 \\
\hline pétrir & 6 & 2 & 1,90 & 7,16 & 3048,47 & 261,57 & 5,68 & 3,95 \\
\hline pomper & 6 & 2 & 0,65 & 30,83 & 7159,15 & 680,84 & 5,04 & 3,50 \\
\hline prier & 5 & 2 & 13,26 & 68,43 & 5546,77 & 684,23 & 4,16 & 4,64 \\
\hline ramer & 5 & 2 & 0,97 & 20,93 & 3907,92 & 250,25 & 4,36 & 4,73 \\
\hline ramper & 6 & 2 & 2,90 & 25,53 & 3393,69 & 197,94 & 3,72 & 4,32 \\
\hline raser & 5 & 2 & 4,03 & 30,42 & 5396,94 & 329,42 & 4,52 & 4,50 \\
\hline repasser & 8 & 3 & 5,45 & 19,45 & 3450,72 & 473,68 & 3,92 & 4,68 \\
\hline saluer & 6 & 2 & 10,06 & 39,87 & 4220,67 & 205,51 & 4,48 & 4,09 \\
\hline semer & 5 & 2 & 3,03 & 17,72 & 7081,73 & 399,07 & 4,56 & 4,18 \\
\hline signer & 6 & 2 & 9,23 & 200,88 & 3330,94 & 544,80 & 4,84 & 4,41 \\
\hline tracer & 6 & 2 & 8,16 & 104,65 & 5179,41 & 981,68 & 3,88 & 3,82 \\
\hline trier & 5 & 2 & 2,90 & 7,13 & 4451,79 & 268,82 & 4,24 & 4,14 \\
\hline vêtir & 5 & 2 & 2,06 & 42,43 & 2100,48 & 93,04 & 5,16 & 3,64 \\
\hline
\end{tabular}


Words used in Experiment 2

\begin{tabular}{|c|c|c|c|c|c|c|c|c|c|}
\hline & ANIMALS & LETT & SYLL & FQ & LEM & BIGR & TRIG & AoA & IMAG \\
\hline \multirow{9}{*}{ 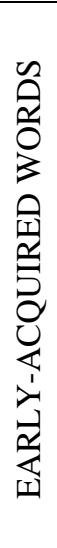 } & butiner & 7 & 3 & 0,19 & 1,11 & 3459,07 & 288,94 & 3,93 & 4,93 \\
\hline & couver & 6 & 2 & 1,13 & 23,42 & 14034,73 & 2327,80 & 4,00 & 4,60 \\
\hline & hiberner & 8 & 3 & 0,06 & 0,27 & 2108,77 & 250,57 & 3,27 & 2,87 \\
\hline & miauler & 7 & 2 & 0,97 & 3,4 & 2436,72 & 163,30 & 2,07 & 5,27 \\
\hline & picorer & 7 & 3 & 0,65 & 2,01 & 4490,46 & 713,79 & 3,13 & 5,00 \\
\hline & pondre & 6 & 1 & 1,13 & 4,2 & 10521,68 & 819,88 & 2,47 & 4,67 \\
\hline & ronronner & 9 & 3 & 1 & 5,61 & 5048,65 & 394,59 & 3,07 & 4,73 \\
\hline & rugir & 5 & 2 & 0,55 & 4,34 & 1138,34 & 14,85 & 3,87 & 5,00 \\
\hline & trotter & 7 & 2 & 1,45 & 6,41 & 4836,18 & 852,82 & 4,40 & 4,47 \\
\hline \multirow{9}{*}{ 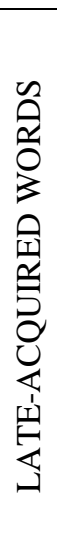 } & bêler & 5 & 2 & 0,13 & 1,23 & 4688,29 & 235,57 & 5,27 & 3,87 \\
\hline & braire & 6 & 1 & 0,39 & 0,48 & 6662,70 & 761,49 & 5,87 & 3,13 \\
\hline & cabrer & 6 & 2 & 0,61 & 3,89 & 5014,34 & 240,29 & 5,00 & 3,47 \\
\hline & glousser & 8 & 2 & 0,68 & 2,93 & 2195,63 & 269,17 & 5,67 & 3,47 \\
\hline & hennir & 6 & 2 & 0,13 & 1,18 & 3206,45 & 54,68 & 4,93 & 4,27 \\
\hline & laper & 5 & 2 & 0,32 & 1,35 & 8757,24 & 145,75 & 6,27 & 3,93 \\
\hline & paître & 6 & 1 & 1,26 & 3,16 & 10739,32 & 1182,09 & 6,33 & 3,67 \\
\hline & parader & 7 & 3 & 0,48 & 10,27 & 8068,34 & 2334,79 & 6,13 & 3,53 \\
\hline & piailler & 8 & 2 & 0,32 & 2,59 & 3566,44 & 540,63 & 6,00 & 3,33 \\
\hline
\end{tabular}

\begin{tabular}{|c|c|c|c|c|c|c|c|c|c|}
\hline & HUMANS & LETT & SYLL & FQ & LEM & BIGR & TRIG & AOA & IMAG \\
\hline \multirow{9}{*}{ 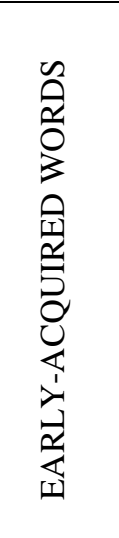 } & agrafer & 7 & 3 & 0,23 & 2,90 & 1835,31 & 68,29 & 4,40 & 6,00 \\
\hline & border & 6 & 2 & 1,19 & 27,84 & 4176,23 & 335,87 & 3,73 & 4,47 \\
\hline & boutonner & 9 & 3 & 0,74 & 4,54 & 7637,80 & 1349,60 & 2,93 & 5,33 \\
\hline & broder & 6 & 2 & 1,26 & 13,64 & 4120,21 & 232,44 & 4,67 & 5,07 \\
\hline & colorier & 8 & 3 & 0,32 & 3,19 & 5898,39 & 615,55 & 1,60 & 5,47 \\
\hline & jongler & 7 & 2 & 0,94 & 2,48 & 6503,16 & 289,80 & 3,13 & 5,60 \\
\hline & peigner & 7 & 2 & 0,81 & 17,06 & 3148,86 & 288,22 & 3,07 & 5,33 \\
\hline & piloter & 7 & 3 & 1,19 & 14,72 & 2867,20 & 220,29 & 3,67 & 4,93 \\
\hline & saler & 5 & 2 & 0,39 & 79,60 & 6306,76 & 471,06 & 2,67 & 5,47 \\
\hline MEAN & & 6,89 & 2,44 & 0,79 & 18,44 & 4721,55 & 430,13 & 3,32 & 5,30 \\
\hline מ & ciseler & 7 & 2 & 0,23 & 4,31 & 3577,12 & 168,44 & 6,53 & 3,20 \\
\hline 0 & émincer & 7 & 3 & 0,10 & 1,19 & 2639,18 & 242,97 & 6,47 & 3,73 \\
\hline a & inciser & 7 & 3 & 0,16 & 1,32 & 4311,07 & 423,84 & 6,60 & 3,53 \\
\hline 号 & malaxer & 7 & 3 & 0,19 & 1,25 & 4093,32 & 355,76 & 5,93 & 4,53 \\
\hline U & mendier & 7 & 2 & 1,81 & 7,92 & 4828,06 & 908,34 & 5,53 & 5,33 \\
\hline $\begin{array}{l}4 \\
4 \\
4\end{array}$ & moudre & 6 & 1 & 0,74 & 2,96 & 11448,39 & 521,15 & 5,60 & 3,80 \\
\hline 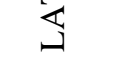 & pétrir & 6 & 2 & 1,90 & 7,16 & 3048,48 & 566,70 & 5,13 & 5,13 \\
\hline
\end{tabular}




\begin{tabular}{|r|r|r|r|r|r|r|r|r|r|}
\hline récurer & 7 & 3 & 0,42 & 1,71 & 3440,21 & 484,71 & 6,00 & 4,47 \\
\hline vernir & 6 & 2 & 0,39 & 16,33 & 3412,81 & 660,46 & 4,93 & 4,53 \\
\hline
\end{tabular}




\section{Bibliography}

Barry, C., Morrison, C. M., \& Ellis, A. W. (1997). Naming the Snodgrass and Vanderwart pictures: Effects of age-of-acquisition, frequency and name agreement. Quarterly Journal of Experimental Psychology: Human Experimental Psychology, 50A, 560-585.

Bogka, N., Masterson, J., Druks, J., Fragkioudaki, M., Chatziprokopiou, E. S. \& Economou, K. (2003). Object and action picture naming in English and Greek. The European Journal of Cognitive Psychology, 15, 371-403.

Bonin P., Fayol, M., \& Chalard, M. (2001). Age of acquisition and word frequency in written picture naming. The Quarterly Journal of Experimental Psychology, 54A(2), 469-489.

Bonin, P., Boyer, B., Méot, A., Fayol, M., \& Droit, S. (2004). Psycholinguistic norms for action photographs in French and their relationships with spoken and written latencies. Behavior Brain Methods, Instruments and Computers, 36(1), 127-139.

Boulenger, V., Roy, A. C., Paulignan, Y., Déprez, V., Jeannerod, M. \& Nazir, T. A. (in press). Cross- talk between language processes and overt motor behavior in the first 200 ms of processing. Journal of Cognitive Neuroscience.

Brown, D. A. \& Watson, F. L. (1987). First in, first out: Word learning age and spoken word frequency as predictors of word familiarity and word naming latency. Memory and Cognition, 15(3), 208-216.

Brysbaert, M. (1996). Word frequency affects naming latency in Dutch when age of acquisition is controlled. European Journal of Cognitive Psychology, 8, 185-193.

Buccino, G., Binkofski, G., Fink, G. R., Fadiga, L., Fogassi, L., Gallese, V., Seitza, R. J., Zilles, G., Rizzolatti, G. \& Freund, H. J. (2001). Action observation activates the premotor and parietal areas in a somatotopic manner: an fMRI study. European Journal of Neuroscience, 13, 400-404.

Buccino, G., Lui, F., Canessa, L., Patteri, I., Lagravinese, G., Benuzzi, F., Porro, C. A. \& Rizzolatti, G. (2004). Neural circuits involved in the recognition of actions performed by nonconspecifics: an fMRI study. Journal of Cognitive Neuroscience, 16(1), 114-126.

Buccino, G., Riggio, L., Melli, G., Binkofski, F., Gallese, V. \& Rizzolatti, G. (2005). Listening to action-related sentences modulates the activity of the motor system: a combined TMS and behavioural study. Brain Research Cognitive Brain Research, 24(3), 355-363.

Caramazza, A., \& Hillis, A.E. (1991). Lexical organization of nouns and verbs in the brain. Nature, 349, 788-790. 
Carroll, J. B., \& White, M. N. (1973a). Age of acquisition norms for 220 picturable nouns. Journal of Verbal Learning and Verbal Behavior, 12, 563-576.

Carroll, J. B., \& White, M. N. (1973b). Word frequency and age-of-acquisition as determiners of picture-naming latency. The Quarterly Journal of Experimental Psychology, 25, 8595.

Colombo, L., \& Burani, C. (2002). The influence of age of acquisition, root frequency and context availability in processing nouns and verbs. Brain and Language, 81, 398-411.

Damasio, A. R., \& Tranel, D. (1993). Nouns and verbs are retrieved with differently distributed neural systems. Proceedings of the National Academy of Science USA, 90, 4957-4960.

Daniele, A., Giustolisi, L., Silveri, M. C., Colosimo, C., \& Gainotti, G. (1994). Evidence for a possible neuroanatomical basis for lexical processing of nouns and verbs. Neuropsychologia, 32(11), 1325-1341.

Ellis, A. W., \& Lambon-Ralph, M. A. (2000). Age of acquisition effects in adult lexical processing reflect loss of plasticity in maturing systems: Insights from connectionist networks. Journal of Experimental Psychology: Learning, Memory and Cognition, 26(5), 1103-1123.

Fadiga, L., Fogassi, L., Pavesi, G. \& Rizzolatti, G. (1995). Motor facilitation during action observation: a magnetic stimulation study. Journal of Neurophysiology, 73, 26082611.

Ghyselinck, M., Lewis, M. B., \& Brysbaert, M. (2004). Age of acquisition and the cumulative frequency hypothesis: a review of the literature and a new multi-task investigation. Acta Psychol., 115(1), 43-67.

Gilhooly, K. J., \& Logie, R. H. (1980). Methods and designs: Age of acquisition, imagery, concreteness, familiarity, and ambiguity measures for 1,944 words. Behaviour Research Methods and Instrumentation, 12, 395-427.

Goldfield, B. A. (2000). Nouns before verbs in comprehension vs. production: the view from pragmatics. Journal of Child Language, 27(3), 501-520.

Hauk, O., Johnsrude, I. \& Pulvermüller, F. (2004a). Somatotopic representation of action words in human motor and premotor cortex. Neuron, 41, 301-307.

Hauk, O. \& Pulvermüller, F. (2004b). Neurophysiological distinction of action words in the fronto-central cortex. Human Brain Mapping, 21, 191-201. 
Hebb, D. O. (1949). The organization of behavior. A neuropsychological theory. New York, Wiley.

Lieberman, P. (2002). On the nature and evolution of the neural bases of human language. Am J Phys Anthropol, Suppl 35, 36-62.

Martin, A., Haxby, J. V., Lalonde, F. M., Wiggs, C. L., \& Ungerleider, L. G. (1995). Discrete cortical regions associated with knowledge of color and knowledge of action. Science, 270(5233), 102-105.

Mason, M. F., Banfield, J. F. \& Macrae, C. N. (2004). Thinking about actions: the neural substrates of person knowledge. Cerebral Cortex, 14, 209-214.

Miceli, G., Silveri, M. C., Villa, G., \& Caramazza, C. (1984). On the basis for the agrammatic's difficulty in producing main verbs. Cortex, 20(2), 207-220.

Monaghan, J. \& Ellis, A. W. (2002). What exactly interacts with spelling-sound consistency in word naming? Journal of Experimental Psychology: Learning, Memory and Cognition, 28, 183-206.

Morrison, C. M., Ellis, A. W., \& Quinlan P. T. (1992). Age of acquisition, not word frequency, affect object naming, not object recognition. Memory and Cognition, 20(6), 705-714.

Morrison, C. M., Chappell, T. D., \& Ellis, A. W. (1997). Age of acquisition norms for a large set of object names and their relation to adult estimates and other variables. The Quarterly Journal of Experimental Psychology: Human Experimental Psychology, 50A, 528-559.

Morrison, C. M., Hirsh, W., \& Duggan, G. B. (2003). Age of acquisition, ageing, and verb production: Normative and experimental data. The Quarterly Journal of Experimental Psychology, 56(4), 705-730.

New, B., Pallier, C., Ferrand, L., \& Matos, R. (2001). Une base de données lexicales du français contemporain sur internet: LEXIQUE, L'Année Psychologique, 101, 417-462.

Oldfield, R. C. (1971). The assessment and analysis of handedness: the Edinburgh Inventory, Neuropsychologia, 9, 97-113.

Oliveri, M., Finocchiaro, C., Shapiro, K., Gangitano, M., Caramazza, A. \& Pascual-Leone, A. (2004). All talk and no action: a transcranial magnetic stimulation study of motor cortex activation during action word production. Journal of Cognitive Neuroscience, 16(3), 374-381.

Perani, D., Cappa, S. F., Schnur, T., Tettamanti, M., Collina, S., Rosa, M. M., \& Fazio, F. (1999). The neural correlates of verb and noun processing. Brain, 122(12), 2337-2344. 
Petersen, S., Fox, P., Posner, M., Mintun, M. \& Raichle, M. (1989). Positron emission tomography studies of the processing of single words. Journal of Cognitive Neuroscience, $1,153-170$.

Preissl, H., Pulvermüller, F., Lutzenberger, W., \& Birbaumer, N. (1995). Evoked potentials distinguish between nouns and verbs. Neuroscience Letters, 197, 81-83.

Pulvermüller, F. (1999a). Words in the brain language. Behavioral Brain Sciences, 22, 253336.

Pulvermüller, F., Lutzenberger, W. \& Preissl, H. (1999b). Nouns and verbs in the intact brain: Evidence from event-related potentials and high-frequency cortical responses. Cerebral Cortex, 9(5), 497-506.

Pulvermüller, F. (2003). The neuroscience of language: On brain circuits of words and serial order, Cambridge University Press.

Pulvermüller, F. (2005a). Brain mechanisms linking language and action. Nature Review Neuroscience, 6(7), 576-582.

Pulvermüller, F., Shtyrov, Y. \& Ilmoniemi, R.J. (2005b). Brain signatures of meaning access in action word recognition. Journal of Cognitive Neuroscience, 17(6), 884-892.

Pulvermüller, F., Hauk, O., Nikulin, V. V. \& Ilmoniemi, R. J. (2005c). Functional links between motor and language systems. European Journal of Neuroscience, 21(3), 793797.

Sereno, S. C. \& Rayner, K. (2003). Measuring word recognition in reading: eye movements and event-related potentials. Trends in Cognitive Sciences, 7(11), 489-493.

Shapiro, K. A., Pascual-Leone, A., Mottaghy, F. M., Gangitano, M., \& Caramazza, A. (2001). Grammatical distinctions in the left frontal cortex. Journal of Cognitive Neuroscience, 13(6), 713-720.

Shapiro, K. A., \& Caramazza, A. (2003). The representation of grammatical categories in the brain. Trends in Cognitive Sciences, 7(5), 201-206.

Shtyrov, Y., Hauk, O. \& Pulvermüller, F. (2004). Distributed neuronal networks for encoding category-specific semantic information: the mismatch negativity to action words.

European Journal of Neuroscience, 19, 1083-1092.

Stadthagen-Gonzalez, H., Bowers, J. S., \& Damian, M. F. (2004). Age of acquisition effects in lexical decision: Evidence from expert vocabularies. Cognition, 93, 11-26.

Tettamanti, M., Buccino, G., Saccuman, M. C., Gallese, V., Danna, M., Scifo, P., Fazio, F., Rizzolatti, G., Cappa, S. F., \& Perani, D. (2005). Listening to action related sentences activates fronto-parietal motor circuits. Journal of Cognitive Neuroscience, 17, 273-281. 
Zevin, J.D., \& Seidenberg, M.S. (2002). Age of acquisition effects in word reading and othertasks. Journal of Memory and Language, 47, 1-29. 
Table 1: Mean values of word frequency, rated AoA, and rated imageability for the 306 words.

\begin{tabular}{|l|c|c|c|}
\hline & NOUN & VERB & $\begin{array}{c}\text { ANOVA } \\
\text { (by item) }\end{array}$ \\
\hline $\begin{array}{l}\text { Word } \\
\text { Frequency }\end{array}$ & 9.13 & 9.35 & $\mathrm{~ns}$ \\
\hline AoA & 4.24 & 3.83 & $\begin{array}{c}\mathrm{F}(1,304)=8.403 ; \\
\mathrm{p}=.004\end{array}$ \\
\hline Imageability & 4.8 & 4.0 & $\begin{array}{c}\mathrm{F}(1,304)=83.88 ; \\
\mathrm{p}<.0001\end{array}$ \\
\hline
\end{tabular}

Figure 1: Upper panel: Mean reaction time for early and late acquired target nouns and verbs plotted as a function of (1a) AoA and (1b) word frequency. Result for the subset of 152 control items is plotted as a function of frequency in (1c). ANOVAs by subjects are reported in the figures $\left(\left(^{*}\right)=\right.$ significant; $(\mathrm{ns})=$ non significant). Lower panel: Corresponding scatterplots with regression analyses, coefficient of determination $\left(\mathrm{R}^{2}\right)$ and $\mathrm{p}$-values. Nouns $=$ black circles; verbs = grey circles.

Figure 2: Left panel: Mean reaction time for verbs denoting actions specific to animals and to humans plotted as a function of AoA $\left(\left(^{*}\right)=\right.$ significant; (ns) = non significant). Right panel: Corresponding scatterplots with regression analyses, coefficient of determination $\left(\mathrm{R}^{2}\right)$ and $\mathrm{p}$ values. Animals: black circles; humans: grey circles. 
Figure 1

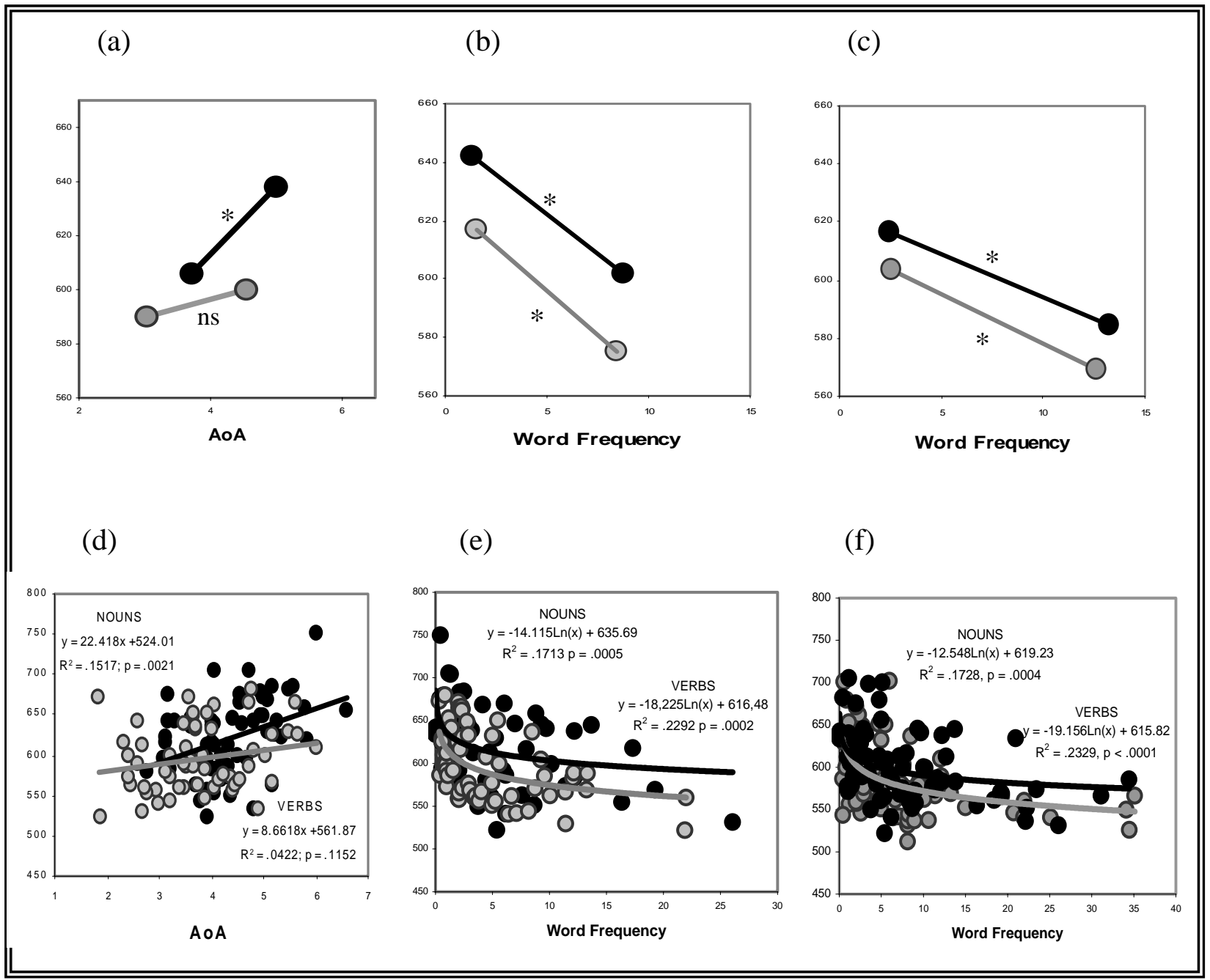

Figure 2

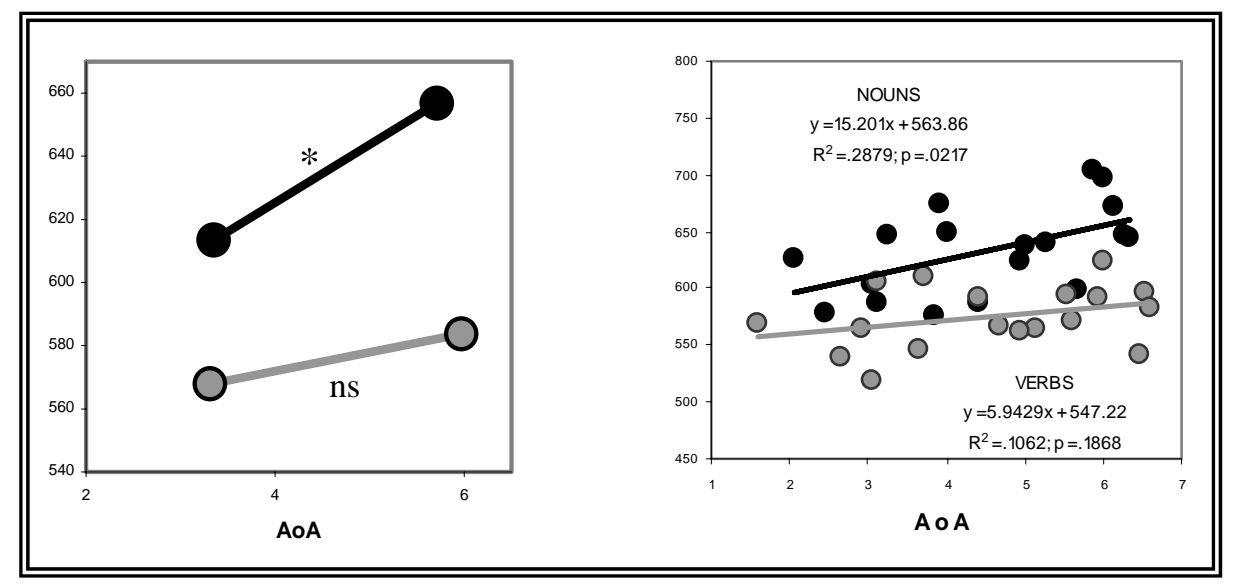




\section{Supplementary Methods 1}

Lexical statistics for the set of 306 words and pseudo-words used in Experiment 1

Mean values of word frequency (FQ), lemma frequency (LEM), length in letters (LETT), bigram frequency (BIGR) and trigram frequency (TRIG), and analyses of variance (ANOVA) by item, for nouns, verbs and pseudo-words.

\begin{tabular}{|l|c|c|c|c|c|c|}
\cline { 2 - 7 } \multicolumn{1}{l|}{} & NOUNS & VERBS & ANOVA (by item) & $\begin{array}{l}\text { PSEUDO- } \\
\text { NOUNS }\end{array}$ & $\begin{array}{l}\text { PSEUDO- } \\
\text { VERBS }\end{array}$ & ANOVA \\
\hline FQ & 9.19 & 9.35 & {$[\mathrm{~F}(1,304)=.010 ; \mathrm{p}=.9196]$} & - & - & - \\
\hline LEM & 14.67 & 58.94 & {$[\mathrm{~F}(1,304)=38.860 ; \mathrm{p}<.0001]$} & - & - & - \\
\hline LETT & 6.34 & 6.434 & $* * * * * * * * * *$ & 6.34 & 6.34 & $* * * * * * * * * * *$ \\
\hline BIGR & 3875 & 4268 & {$[\mathrm{~F}(1,304)=2.915 ; \mathrm{p}=.0888]$} & 4743 & 5308 & {$[\mathrm{~F}(1,304)=2.251 ; \mathrm{p}=.1346]$} \\
\hline TRIG & 543 & 534 & {$[\mathrm{~F}(1,304)=.024 ; \mathrm{p}=.8773]$} & 617 & 530 & {$[\mathrm{~F}(1,304)=1.400 ; \mathrm{p}=.2377]$} \\
\hline
\end{tabular}

Note: lemma frequency could not be matched between the two classes of words, but the simple regression analysis below reveals a high correlation between lemma- and word frequency (which was controlled) for the two word categories.

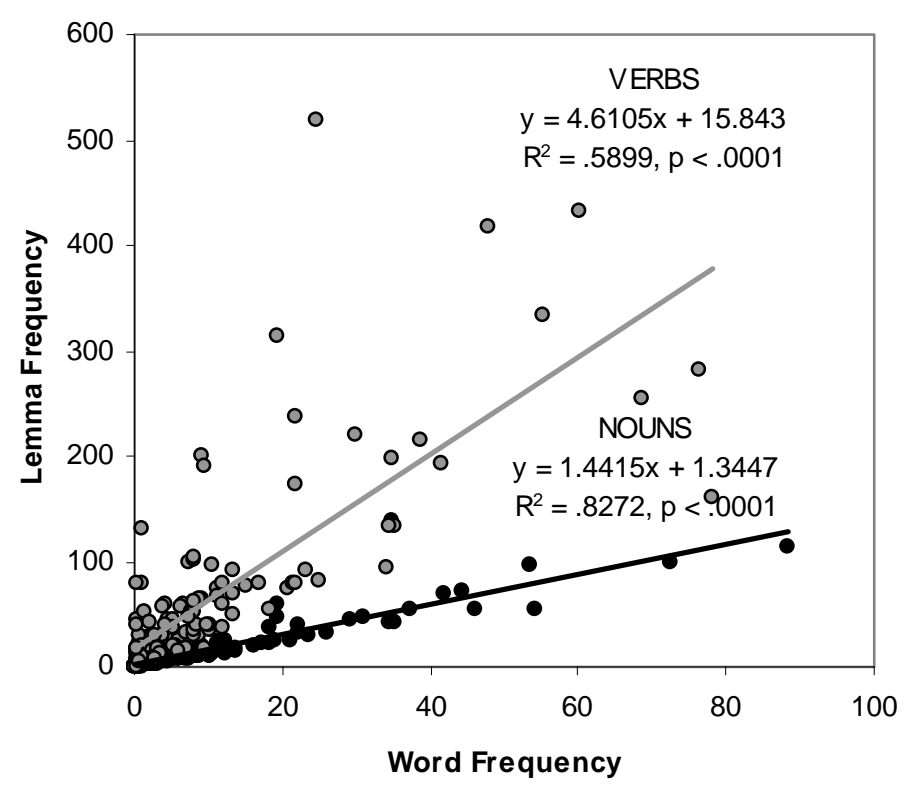

Suppl. Figure 1: Scatterplots of lemma frequency for each of the initial 153 nouns and 153 verbs as a function of word frequency. Regression analyses, coefficient of determination $\left(\mathrm{R}^{2}\right)$ and $\mathrm{p}$-values are reported for each grammatical category. Nouns = black circles; verbs = grey circles. 


\section{Supplementary Methods 2}

Lexical statistics for the set of target and control words used in Experiment 1

Mean values of AoA, word frequency (FQ), lemma frequency (LEM), imageability (IMAG), length in letters (LETT), bigram frequency (BIGR), trigram frequency (TRIG) and number of syllables (SYLL) for nouns and verbs with different mean AoA (a) and different mean frequency (b and c).

(a) Target words.

\begin{tabular}{|c|c|c|c|c|c|c|c|c|}
\hline NOUN & AoA & FQ & LEM & IMAG & LETT & BIGR & TRIG & SYLL \\
\hline $\begin{array}{l}\text { Early } \\
(\mathrm{n}=30)\end{array}$ & 3.71 & 5.07 & 9 & 4.92 & 6.26 & 3617 & 533 & 2 \\
\hline $\begin{array}{c}\text { Late } \\
(\mathrm{n}=30)\end{array}$ & 5.02 & 5.07 & 8 & 4.62 & 6.06 & 3931 & 609 & 2 \\
\hline Statistics & $\begin{array}{c}{[F(1,58)=} \\
107,68 ; \mathbf{p}< \\
.0001]\end{array}$ & ns & ns & ns & ns & ns & ns & ns \\
\hline VERB & AoA & FQ & LEM & IMAG & $\begin{array}{l}\text { LETT } \\
\end{array}$ & BIGR & TRIG & SYLL \\
\hline $\begin{array}{l}\text { Early } \\
(\mathrm{n}=30)\end{array}$ & 3.03 & 5.02 & 34.71 & 4.23 & 6.66 & 3711 & 496 & 2.37 \\
\hline $\begin{array}{c}\text { Late } \\
(\mathrm{n}=30)\end{array}$ & 4.54 & 4.7 & 42 & 4.08 & 6.13 & 4675 & 470 & 2 \\
\hline Statistics & $\begin{array}{c}F(1,58)= \\
98.397 ; \mathbf{p}< \\
.0001]\end{array}$ & ns & ns & ns & ns & ns & ns & ns \\
\hline
\end{tabular}

(b) The same words as in (a) when grouped as a function of word frequency.

\begin{tabular}{|c|c|c|c|c|c|c|c|c|}
\hline NOUN & AoA & FQ & LEM & IMAG & LETT & BIGR & TRIG & SYLL \\
\hline Low $(n=30)$ & 4.39 & 1.34 & 2.89 & 4.85 & 5.86 & 3023 & 316 & 2.1 \\
\hline High $(n=30)$ & 4.34 & 8.79 & 13.81 & 4.68 & 6.46 & 4524 & 825 & 2 \\
\hline Statistics & ns & $\begin{array}{c}{[F(1,58)=} \\
55.938 ; p \\
<.0001]\end{array}$ & $\begin{array}{c}{[F(1,58)=} \\
39.600 ; p< \\
.0001]\end{array}$ & ns & $\begin{array}{c}{[F(1,58)=} \\
6.4005 ; p \\
=.0141]\end{array}$ & $\begin{array}{c}{[F(1,58)=} \\
63.869 ; p \\
=.0142]\end{array}$ & $\begin{array}{c}{[F(1,58)=} \\
10.593 ; p \\
=.0019]\end{array}$ & ns \\
\hline VERB & AoA & FQ & LEM & IMAG & LETT & BIGR & TRIG & SYLL \\
\hline Low $(n=30)$ & 4 & 1.44 & 19.13 & 4.17 & 6.46 & 4004 & 449 & 2.26 \\
\hline High $(n=30)$ & 3.56 & 8.27 & 57.90 & 4.14 & 6.33 & 4381 & 515 & 2.3 \\
\hline Statistics & ns & $\begin{array}{c}\mathrm{F}(1,58)= \\
\mathbf{5 6 . 6 3 4 ;} \mathrm{p} \\
<.0001]\end{array}$ & $\begin{array}{c}{[F(1,58)=} \\
11.975 ; p= \\
.0011]\end{array}$ & ns & ns & ns & ns & ns \\
\hline
\end{tabular}

(c) The 152 control-words.

\begin{tabular}{|c|c|c|c|c|c|c|c|c|}
\hline NOUN & AoA & FQ & LEM & IMAG & LETT & BIGR & TRIG & SYLL \\
\hline Low $(n=38)$ & 3.6 & 2.5 & 4.8 & 5.2 & 6.3 & 4036 & 631 & 2 \\
\hline High $(n=38)$ & 3.6 & 13.2 & 19.8 & 5.2 & 6.7 & 4229 & 586 & 2 \\
\hline Statistics & ns & $\begin{array}{c}{[F(1,74)=} \\
125.95 ; p< \\
.0001]\end{array}$ & $\begin{array}{c}{[F(1,74)=} \\
65.52 ; p< \\
.0001]\end{array}$ & ns & ns & ns & ns & ns \\
\hline VERB & AoA & FQ & LEM & IMAG & LETT & BIGR & TRIG & SYLL \\
\hline Low $(n=38)$ & 3.7 & 2.5 & 24 & 4.1 & 6.4 & 3877 & 390 & 2.3 \\
\hline High $(n=38)$ & 3.5 & 12.6 & 72.4 & 4.1 & 6.6 & 4263 & 507 & 2.2 \\
\hline Statistics & ns & $\begin{array}{c}{[F(1,74)=} \\
53.65 ; p< \\
.0001]\end{array}$ & $\begin{array}{c}{[F(1,74)=} \\
21.39 ; p< \\
.0001]\end{array}$ & ns & ns & ns & ns & ns \\
\hline
\end{tabular}




\section{Supplementary Methods 3}

\section{Methods and Material used in Experiment 2}

Twenty volunteers, aged from 19 to 29 years old, participated in this experiment. All were native French speakers, and had normal or corrected to normal vision. Right-handedness was determined with the Edinburgh inventory (scores between 0.56 and 0.88; Oldfield, 1971). None of the participants took part in the first experiment.

Mean values of word frequency (FQ), lemma frequency (LEM), length in letters (LETT), bigram frequency (BIGR), trigram frequency (TRIG), number of syllables (SYLL), and analyses of variance (ANOVA) by item, for 36 verbs related to actions specific to animals $(\mathrm{n}=18)$ and humans $(\mathrm{n}=18)$, and for matched pseudo-words $(\mathrm{n}=36)$.

\begin{tabular}{|l|c|c|c|c|c|}
\hline & ANIMALS & HUMANS & ANOVA (by item) & $\begin{array}{c}\text { PSEUDO- } \\
\text { WORDS }\end{array}$ & ANOVA \\
\hline FQ & .63 & .72 & $\begin{array}{c}{[\mathrm{F}(1,34)=.2730 ; \mathrm{p}} \\
=.6047]\end{array}$ & - & - \\
\hline LEM & 4.32 & 11.67 & $\begin{array}{c}{[\mathrm{F}(1,34)=2.6220 ;} \\
\mathrm{p}=.1146]\end{array}$ & - & - \\
\hline LETT & 6.61 & 6.77 & $\begin{array}{c}{[\mathrm{F}(1,34)=.2402 ; \mathrm{p}} \\
=.6212]\end{array}$ & 6.69 & $* * * * * *$ \\
\hline BIGR & 5609 & 4627 & $\begin{array}{c}{[\mathrm{F}(1,34)=.9896 ; \mathrm{p}} \\
=.3269]\end{array}$ & 4822 & $\begin{array}{c}{[\mathrm{F}(1,70)=.2360 ;} \\
\mathrm{p}=.6286]\end{array}$ \\
\hline TRIG & 673 & 455 & $\begin{array}{c}{[\mathrm{F}(1,34)=1.4499 ;} \\
\mathrm{p}=.2371]\end{array}$ & 526 & $\begin{array}{c}{[\mathrm{F}(1,70)=.0498 ;} \\
\mathrm{p}=.8240]\end{array}$ \\
\hline SYLL & 2.1 & 2.4 & $\begin{array}{c}{[\mathrm{F}(1,34)=1.6798 ;} \\
\mathrm{p}=.2037]\end{array}$ & 2.25 & $* * * * * *$ \\
\hline
\end{tabular}

Mean values of AoA, word frequency (FQ), lemma frequency (LEM), imageability (IMAG), length in letters (LETT), bigram frequency (BIGR), trigram frequency (TRIG) and number of syllables (SYLL) for verbs designating actions specific to animals and to humans with different mean AoA. Word age of acquisition was controlled with empirical ratings of 15 subjects on a seven-point scale (according to Gilhooly \& Logie, 1980; $1=$ [0-2] and $7=$ [older than 13 years old]). Word imageability was evaluated in the same way by another group of 15 subjects (with $0=$ impossible and $6=$ very easy to generate a mental image of the word). Note that imageability could not be matched between early and late-acquired items for the two conditions. However, it was higher for early than for late-acquired items for both subcategories of verbs (difference of 1 and 1.11, respectively).

\begin{tabular}{|c|c|c|c|c|c|c|c|c|}
\hline ANIMALS & AoA & FQ & LEM & IMAG & LETT & BIGR & TRIG & SYLL \\
\hline Early $(n=9)$ & 3.35 & 0.79 & 5.64 & 4.61 & 6.88 & 5341 & 647 & 2.33 \\
\hline Late $(n=9)$ & 5.71 & 0.48 & 3.01 & 3.62 & 6.33 & 5877 & 641 & 1.88 \\
\hline Statistics & $\begin{array}{c}{[F(1,16)=} \\
56.56 ; p< \\
.0001]\end{array}$ & ns & ns & $\begin{array}{r}{[F(1,16)=} \\
14.35 ; p= \\
.0016]\end{array}$ & $\mathrm{ns}$ & ns & ns & ns \\
\hline HUMANS & AoA & FQ & LEM & IMAG & $\begin{array}{l}\text { LETT } \\
\end{array}$ & BIGR & TRIG & SYLL \\
\hline Early $(n=9)$ & 3.31 & 0.78 & 18.44 & 5.29 & 6.88 & 4721 & 430 & 2.44 \\
\hline Late $(n=9)$ & 5.97 & 0.73 & 3.73 & 4.18 & 6.71 & 4533 & 481 & 2.28 \\
\hline Statistics & $\begin{array}{c}{[F(1,16)=} \\
47.14 ; p< \\
.0001]\end{array}$ & ns & ns & $\begin{array}{c}{[F(1,16)=} \\
13.62 ; p= \\
.0020]\end{array}$ & ns & ns & ns & ns \\
\hline
\end{tabular}




\section{Supplementary Results}

\section{Multiple regressions analyses for words used in Experiment 1.}

Multiple regressions with hierarchical analysis using mean reaction time as dependent variable and the seven following independent variables ${ }^{1}$ (bigram frequency (BIGR), trigram frequency (TRIG), number of syllables (SYLL), length in letters (LETT), imageability (IMAG), word frequency ( $\log _{10}$ Fq) and age of acquisition (AoA)) are presented for (a) the entire set of 306 words, (b) the 2*60 early and late acquired target nouns and target verbs, and (c) the subset of 152 items. Coefficient of determination $\left(R^{2}\right)$ calculated after each variable has been entered into the analysis, together with coefficient of regression (Beta) and p-values (t-test) corresponding to each variable, are reported for nouns and verbs. \% of additional variance explained by each variable is also reported for each word category.

(a)

\begin{tabular}{|l|c|c|c|c|c|c|c|c|}
\cline { 2 - 9 } \multicolumn{1}{c|}{} & \multicolumn{4}{c|}{ NOUNS } & \multicolumn{4}{c|}{ VERBS } \\
\cline { 2 - 9 } \multicolumn{1}{c|}{} & $\mathrm{R}^{2}$ & Beta & $\mathrm{p}$ & $\begin{array}{c}\text { \% of } \\
\text { additional } \\
\text { variance }\end{array}$ & $\mathrm{R}^{2}$ & Beta & $\mathrm{p}$ & $\begin{array}{c}\text { \% of } \\
\text { additional } \\
\text { variance }\end{array}$ \\
\hline BIGR & .0013 & .0958 & .2270 & & .0025 & .0668 & .4624 & \\
\hline TRIG & .0174 & -.1002 & .2385 & 1.6 & .0170 & -.0283 & .7582 & 1.4 \\
\hline SYLL & .0180 & -261 & .7157 & .06 & .0173 & -.0090 & .9215 & .03 \\
\hline LETT & .0354 & -.0749 & .3038 & 1.7 & .0174 & .0696 & .4381 & .01 \\
\hline IMAG & .2243 & -.0388 & .7003 & 18.9 & .0681 & -.1272 & .1556 & 5.07 \\
\hline Log 10 FQ & .4131 & -.3674 & $\mathbf{. 0 0 0 1}$ & $\mathbf{1 9}$ & .2875 & -.4111 &. $\mathbf{. 0 0 0 1}$ & $\mathbf{2 2}$ \\
\hline AOA & .4681 & .3890 & $\mathbf{. 0 0 0 1}$ & $\mathbf{5 . 5}$ & .2992 & .1646 & .1211 & 1.1 \\
\hline
\end{tabular}

(b)

\begin{tabular}{|l|c|c|c|c|c|c|c|c|}
\cline { 2 - 9 } \multicolumn{1}{c|}{} & \multicolumn{4}{c|}{ NOUNS } & \multicolumn{4}{c|}{ VERBS } \\
\cline { 2 - 9 } \multicolumn{1}{c|}{} & $\mathrm{R}^{2}$ & Beta & $\mathrm{p}$ & $\begin{array}{c}\text { \% of } \\
\text { additional } \\
\text { variance }\end{array}$ & $\mathrm{R}^{2}$ & Beta & $\mathrm{p}$ & $\begin{array}{c}\text { \% of } \\
\text { additional } \\
\text { variance }\end{array}$ \\
\hline BIGR & .0019 & .2506 & .0945 & & .0067 & -.0812 & .5743 & \\
\hline TRIG & .0654 & -.2354 & .1272 & 6.35 & .0085 & .0086 & .9498 & .18 \\
\hline SYLL & .0666 & .0452 & .7179 & .12 & .0135 & -.0172 & .9150 & .5 \\
\hline LETT & .0925 & .0301 & .8231 & 2.59 & .0388 & .2578 & .1067 & 2.53 \\
\hline IMAG & .1223 & -.1069 & .4087 & 2.98 & .0457 & .0822 & .5324 & .69 \\
\hline Log $_{10}$ FQ & .3243 & -.4978 & $\mathbf{. 0 0 0 1}$ & $\mathbf{2 0 . 2}$ & .2343 & -.3857 & $\mathbf{. 0 0 3 9}$ & $\mathbf{1 8 . 8 6}$ \\
\hline AOA & .4153 & .3473 & $\mathbf{. 0 0 6 3}$ & $\mathbf{9 . 1}$ & .2645 & .2068 & .1497 & 3.02 \\
\hline
\end{tabular}

(c)

\begin{tabular}{|l|c|c|c|c|c|c|c|c|}
\cline { 2 - 9 } \multicolumn{1}{c|}{} & \multicolumn{4}{c|}{ NOUNS } & \multicolumn{4}{c|}{ VERBS } \\
\cline { 2 - 9 } \multicolumn{1}{c|}{} & $\mathrm{R}^{2}$ & Beta & $\mathrm{p}$ & $\begin{array}{c}\text { \% of } \\
\text { additional } \\
\text { variance }\end{array}$ & $\mathrm{R}^{2}$ & Beta & $\mathrm{p}$ & $\begin{array}{c}\text { \% of } \\
\text { additional } \\
\text { variance }\end{array}$ \\
\hline BIGR & .0024 & .1493 & .2778 & & .0046 & -.0541 & .6895 & \\
\hline TRIG & .0287 & -.1934 & .1904 & 2.63 & .0241 & .0781 & .5681 & 1.95 \\
\hline SYLL & .0318 & -.1323 & .2922 & .31 & .0327 & .0618 & .6370 & .86 \\
\hline LETT & .0431 & .0193 & .8794 & 1.13 & .0440 & -.0485 & .7172 & 1.13 \\
\hline IMAG & .0593 & -.0193 & .8833 & 1.62 & .0797 & -.1996 & .4095 & 3.57 \\
\hline Log 10 FQ & .2300 & -.4335 &. $\mathbf{0 0 0 1}$ & $\mathbf{1 7 . 0 7}$ & .2893 & -.4563 & $\mathbf{. 0 0 0 1}$ & $\mathbf{2 0 . 9 6}$ \\
\hline AOA & .2683 & .2543 & .0634 & 3.83 & .3070 & .1773 & .1915 & 1.77 \\
\hline
\end{tabular}

\footnotetext{
${ }^{1}$ Note that we included word- rather than lemma frequency as independent variable, since the simple regression analyses for the set of 306 words showed stronger effect of word- than of lemma frequency on performance for the two word categories.
} 
Correlation analyses for words used in Experiment 1.

Correlations among the relevant variables for (a) the entire set of 306 words, (b) the subset of $2 * 60$ early and later-acquired nouns and verbs, and (c) the subset of 152 low and higher frequency words.

(a)

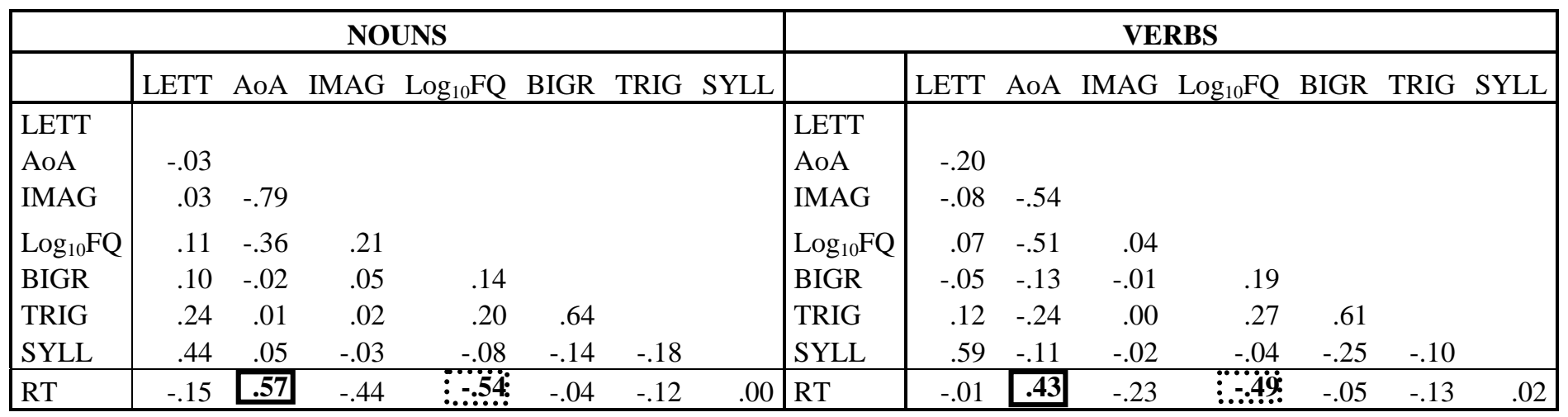

(b)

\begin{tabular}{|c|c|c|c|c|c|c|c|c|c|c|c|c|c|c|c|}
\hline \multicolumn{8}{|c|}{ NOUNS } & \multicolumn{8}{|c|}{ VERBS } \\
\hline & LETT & AoA & IMAG & $\log _{10} \mathrm{FQ}$ & BIGR & TRIG & SYLL & & LETT & AоA & IMAG & $\log _{10} \mathrm{FQ}$ & BIGR & TRIG & SYLL \\
\hline $\begin{array}{l}\text { LETT } \\
\text { AoA }\end{array}$ & -.12 & & & & & & & $\begin{array}{l}\text { LETT } \\
\text { AoA }\end{array}$ & -.25 & & & & & & \\
\hline IMAG & -.20 & -.43 & & & & & & IMAG & -.07 & -.29 & & & & & \\
\hline $\log _{10} \mathrm{FQ}$ & .36 & .00 & -.28 & & & & & $\log _{10} \mathrm{FQ}$ & .08 & -.26 & -.17 & & & & \\
\hline BIGR & .11 & -.04 & .00 & .23 & & & & BIGR & -.24 & .19 & .00 & -.03 & & & \\
\hline TRIG & .19 & -.04 & -.06 & .28 & .69 & & & TRIG & .00 & -.13 & .01 & .06 & .43 & & \\
\hline SYLL & .38 & -.01 & .08 & -.04 & -.19 & -.21 & & SYLL & .63 & -.20 & -.04 & -.09 & -.38 & -.18 & \\
\hline $\mathrm{RT}$ & -.17 & \begin{tabular}{|l|}
.39 \\
\end{tabular} & -.10 & $47 \vdots$ & -.04 & -.21 & .07 & RT & .18 & \begin{tabular}{|l|}
.21 \\
\end{tabular} & .07 & :430 & $\begin{array}{ll}\vdots & -.08 \\
\end{array}$ & -.07 & .10 \\
\hline
\end{tabular}

(c)

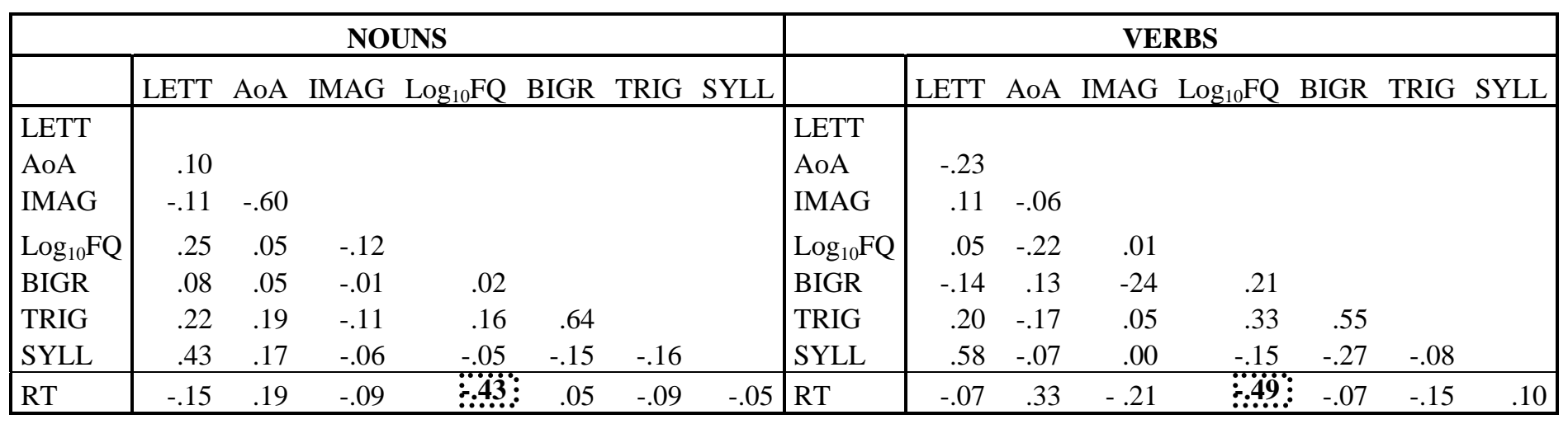

